\title{
An Experience with an Outbreak of Kala-azar in a Rural Area of Bangladesh
}

\author{
Abdur Rahim, ${ }^{1}$ Md. Moniruzzaman, ${ }^{2}$ Mostofa Kamal, ${ }^{3}$ Shah Golam Nabi, ${ }^{4}$ Supriya Sarkar, ${ }^{5}$ Md. Mujibur Rahman ${ }^{6}$
}

\begin{abstract}
:
Background: A large number of kala-azar cases from a particular village in Debigonj upazila of Panchaghar, a remote district of Bangladesh, led us to carry out the study of the situation.

Materials \& methods: This cross-sectional study was done among clinically screened people at the health camp in two days in Dararhat in Debigonj upazila of Panchaghar.

Results: Around 700 people were screened at the health camp and 51 (7.2\%) were suspected to have either Kala-azar or related disorders where 42 (82\%) were rk-39 test positive. The disease was more prevalent among adult (59.6\%) and children (23.8\%) under the age of 10 years. However, there was no clear linear relationship between the prevalence of the disease and age group. Kala-azar occurred in both male and female, and its prevalence did not correlate significantly with income. A history of fever (73.8\%) and splenomegaly (45.2\%) was noted in most of the kala-azar cases. A significant number of PKDL (26.2\%) cases were also found in that area.
\end{abstract}

Conclusions: Since there are a significant proportion of PKDL cases, eradication will be very tough unless the health authority take adequate measure to identify and treat them properly.

Keywords: Kala-azar, Visceral leishmaniasis, PKDL, rK-39 test.

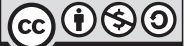

DOI: http://dx.doi.org/l0.3329/jom.v20il.388I7

Copyright: () 2019 Rahim A et al. This is an open access article published under the Creative Commons Attribution-NonCommercial-NoDerivatives 4.0 International License, which permits use, distribution and reproduction in any medium, provided the original work is properly cited, is not changed in any way and it is not used for commercial purposes.

Received: 28 February, 2018;

Accepted: 17 May, 2018

\section{Introduction:}

The parasitic disease kala-azar (visceral Leishmaniasis, VL) was first described in 1824, in Jessore district of greater Bengal, reportedly killed around 75,000 people from 1824 to 1827. ${ }^{1}$ Kala-azar epidemic peaks were recorded in Bengal in the $1820 \mathrm{~s}, 1860 \mathrm{~s}, 1920 \mathrm{~s}$, and 1940s. ${ }^{2,3}$ Historical records describe the classical picture of kala-azar as prolonged irregular fever, progressive emaciation, and enlargement of the liver and spleen. ${ }^{1-3}$ The infection can provoke one of three clinical forms known as cutaneous, visceral, or muco-

1. Resident Physician, Shaheed Suhrawardy Medical College Hospital, Dhaka.

2. OSD, DGHS, Dhaka.

3. Junior consultant of Medicine, Shaheed Suhrawardy Medical College Hospital, Dhaka.

4. Vice- Principal, Mugda Medical College, Dhaka.

5. Deputy Program Manager, Hospital Services, DGHS, Dhaka.

6. Professor, Department of Medicine, Dhaka Medical College, Dhaka.

Corresponding author: Prof. Md. Mujibur Rahman, Department of Medicine, Dhaka Medical College, Dhaka. Mob: +8801711525406, Email: mmrahman61@gmail.com. cutaneous depending on the Leishmania species and the host immune response. ${ }^{4}$ Leishmaniasis is endemic in about 90 countries; at least 12 million people are reported to be infected worldwide, and 400,000 new cases occur each year. ${ }^{5}$ More than $90 \%$ of all cases of VL occur in Bangladesh, India, Nepal, Sudan, and Brazil. ${ }^{6}$ An intensive control program aimed at the eradication of malaria was mounted in the 1950s and 1960s throughout this subcontinent and as a result simultaneous drop in kala-azar incidence was widely seen. ${ }^{7}$ In Bangladesh, sporadic kala-azar cases were reported in the 1970s, and an outbreak occurred in Pabna district in $1980 .{ }^{8}$ Surveillance data are lacking from this period, but in a series of 59 kala-azar patients reported from 1968-1980, reported from at least 7 districts in Bangladesh. ${ }^{9}$ From 1994 to 2004, a total of 73,467 kala-azar cases were reported to the malaria and vector-borne disease control unit of Directorate General of Health Services (DGHS), Government of Bangladesh. ${ }^{7}$ Kala-azar and post kala-azar dermal leishmaniasis (PKDL) are a significant health problem and has been reported from 26 out of 64 districts of the country. ${ }^{10}$ Here we report an outbreak of kala-azar occurred in 2011 in a small village named Dararhat in Debigonj upazila of Panchaghar, a remotest district of Bangladesh. 


\section{Materials \& methods:}

A team from disease control unit of DGHS, went Dararhat to help the local authority of Debigonj upazila health complex. Two health camps were arranged in a community clinic for two consecutive days. Miking was done to ask the people with the history of fever for more than 2 weeks, abdominal mass and past history of kala azar to come to the community clinic of that village. After taking history and clinical examination, suspected cases were requested to attend Debigonj upazila health complex and here rk-39 based screening test was done. Based on the national guideline, the following case definition criteria were used to diagnose the cases:

Kala-azar (KA): History of fever for more than 2 weeks; residing/ traveling in endemic areas; any one of the following symptoms and signs: splenomegaly, weight loss, anemia, and 'rk-39' based ICT test positivity.

PKDL: Residing / travelling in the endemic areas; history of treatment for Kala-azar any time in the past; suggestive skin lesion without loss of sensation, which may be macular, papular, nodular or mixed; exclusion of other causes of skin disease eg. leprosy, vitiligo, pityriasis, ring worm and ' $r k-39$ ' based ICT / Slit skin smear/ PCR positive.

Kala-azar Treatment Failure (KATF): An individual, who is diagnosed to have KA with the above mentioned case definition and history of treatment for KA within last one year, will be reported as KATF.

Relapse Kala-azar (RKA): An individual who is diagnosed to have KA with the above mentioned case definition and history of treatment for KA anytime in the past but not within last one year will be reported as RKA.

Para kala-azar dermal leishmaniasis: There is no specific case definition. Sometimes PKDL may co-exist with VL and patients with PKDL and concomitant VL may be more appropriately referred to as para kala-azar dermal leishmaniasis.

\section{Results:}

Around 700 people were clinically screened at the health camp in two days where $51(7.2 \%)$ were suspected to have either Kala-azar or related disorders. Out of 51 cases 42 (82\%) were rk-39 test positive. Among 42 cases 33 (78.5\%) were male and majority of the cases were in children below the age of 10 years $(23.8 \%)$ and adult $(59.6 \%)$ age group. The occurrence of leishmaniasis was highest among adult males whose primary occupations were day laborer (19\%) and farmer (16.7\%) and most of the females were housewives (14.3\%). Among the cases 19 (45.2\%) did not use sanitary latrine and $38(90.5 \%)$ used mosquito nets but only $24(57.2 \%)$ used regularly (table I).

Table I: Socio-demographic profile of the cases $(n=42)$

\begin{tabular}{|c|c|c|c|}
\hline Characteristics & Group & Sub group & $\begin{array}{c}\text { Frequency } \\
(\%)\end{array}$ \\
\hline \multirow[t]{6}{*}{ Age (year) } & $<10$ & & $10(23.8)$ \\
\hline & $11-20$ & & $7(16.7)$ \\
\hline & $21-30$ & & $13(31.0)$ \\
\hline & $31-40$ & & $5(11.9)$ \\
\hline & $41-50$ & & $4(9.5)$ \\
\hline & $>50$ & & $3(7.1)$ \\
\hline \multirow[t]{2}{*}{ Sex } & Male & & $33(78.5)$ \\
\hline & Female & & $9(21.5)$ \\
\hline \multirow[t]{6}{*}{ Occupation } & House wife & & $6(14.3)$ \\
\hline & Day laborer & & $8(19.0)$ \\
\hline & Farmer & & $7(16.7)$ \\
\hline & Student & & $9(21.4)$ \\
\hline & Dependent & & $10(23.8)$ \\
\hline & Others & & $2(4.8)$ \\
\hline \multirow[t]{3}{*}{ Uses of mosquito net } & Yes & Regular & $24(57.2)$ \\
\hline & & Occasional & $14(33.3)$ \\
\hline & No & & $4(9.5)$ \\
\hline \multirow[t]{2}{*}{ Uses of sanitary latrine } & Yes & & $23(54.8)$ \\
\hline & No & & $19(45.2)$ \\
\hline
\end{tabular}

Nature of their houses was kacha (both wall and floor made of mud) but roofs were tin shaded (Figure $1 \& 2$ ). Fever was present in $31(73.8 \%)$, splenomegaly in $19(45.2 \%)$, hepatosplenomegaly in $8(19.0 \%)$, maculo-papular skin lesion in $13(31.0 \%)$ cases and $21(50.0 \%)$ of them had previous history of kala-azar. Among the 42 cases, 21 (50.0\%) were diagnosed as kala-azar, 11 (26.2\%) were suspected as PKDL (as slit skin smear was not available there), 5 (11.9\%) were treatment failure kala-azar, 3 (7.1\%) were relapsed cases and 2 cases were suspected to have Para Kala-azar dermal leishmaniasis (table II). We started treatment according to our national guidelines and the cases were followed up by the MODC (medical officer disease control) of that health complex. 
Table II: Distribution of the clinical features and diagnosis of the cases $(n=42)$

\begin{tabular}{llc}
\hline Characteristics & Group & Frequency (\%) \\
\hline Clinical features & Fever $>2$ weeks & $31(73.8)$ \\
& Anaemia & $37(88.1)$ \\
& Splenomegaly & $19(45.2)$ \\
& Hepato-splenomegaly & $8(19.0)$ \\
& Maculo-papular skin lesion & $13(31.0)$ \\
& Previous H/O Kala-azar & $21(50.0)$ \\
Diagnosis & Kala-azar & $21(50.0)$ \\
& PKDL (suspected) & $11(26.2)$ \\
& KAFT (Kala-azar failure of treatment) & $5(11.9)$ \\
& Kala-azar relapse & $3(7.1)$ \\
& Para Kala-azar DermalLeishmaniasis (suspected) & $2(4.8)$ \\
\hline
\end{tabular}

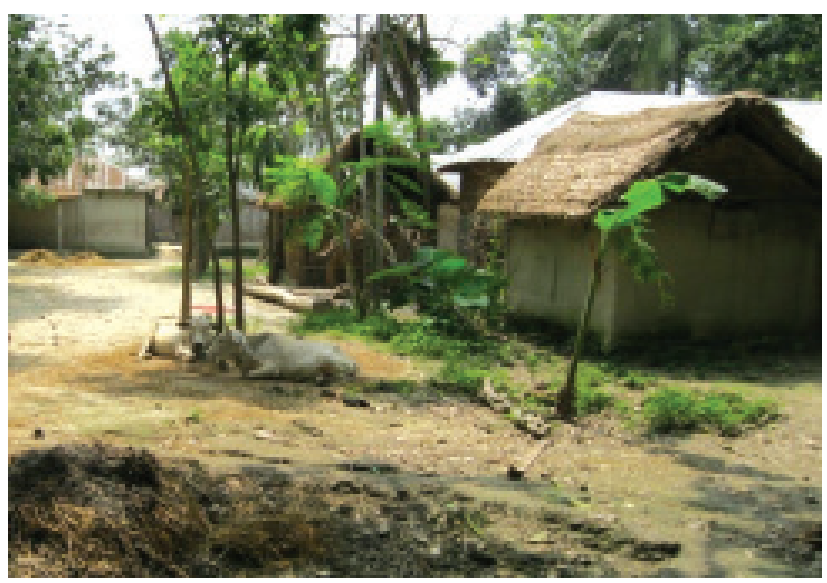

Figure 1: View of Dararhat village

\section{Discussion:}

In the past, kala-azar had been endemic in Bangladesh and the neighbouring parts of India. Epidemics used to occur almost regularly every $15-20$ years. ${ }^{11}$ During the past few years, an increasing number of cases of kala-azar have been reported in various parts of Bangladesh. The detection of large number of clinically and rK-39 test positive kala-azar cases in the present study may be due to a population influx from adjoining areas or to active disease transmission, or both. Since we found no evidence for population movements in the study village, the cases must have been due to a high level of disease transmission. The majority of the cases of our study were adult and children under the age of 10 years, which is in contrast to the findings of Rahman \& Islam ${ }^{9}$, where all the cases were adult as the study was done in Dhaka city and the cases were referred to them by physicians

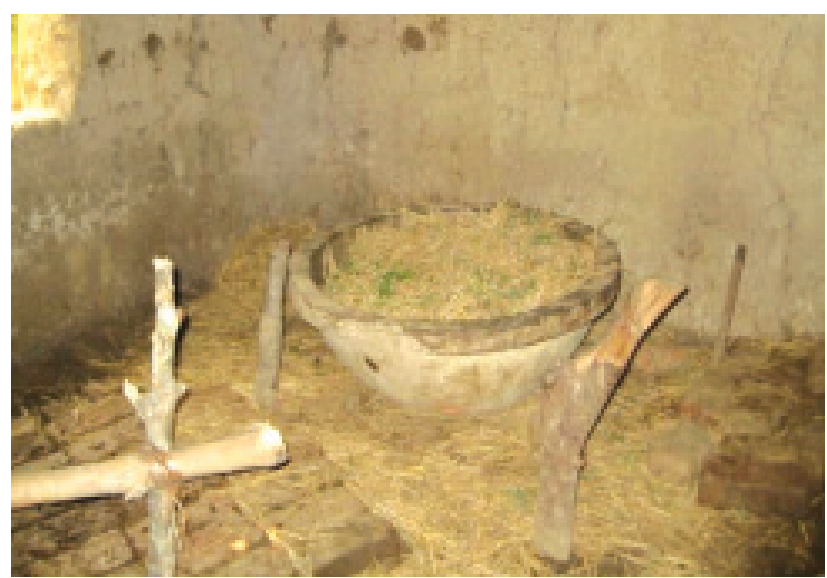

Figure 2: Living place of Sand fly

from different parts of Bangladesh but our study was carried out in the community level. No direct relationship between the occurrence of kala-azar and age group was observed, but in our study the higher prevalence among males $(78.5 \%)$ may be due to the greater exposure to the vector, because of their clothing pattern and habits. The study of Rahman \& Islam $^{9}$ showed $70 \%$ cases were male. African kala-azar has, however, been reported to affect four times as many males as females. ${ }^{12}$ Overall the patterns of occupation of our cases suggest that all of them belongs to poor socio-economic condition and their house is made of mud which is also the residing place of kala-azar vector, sandfly. A good number of cases $(33.3 \%)$ do not use mosquito nets regularly which may be a factor for occurrence of kala-azar in this population. Similar findings also observed in a study by Argaw D et al. ${ }^{6}$ in Ethiopia. We also found a good number of PKDL cases in 
that village which may serve as reservoir of kala-azar and we think which may be responsible for that massive outbreak. We found 2 unusual cases of concomitant PKDL and VL in the community which is recently termed as para kala-azar dermal leishmaniasis. A series of 9 cases described by Kumar et $\mathrm{al}^{13}$ indicates that para kala-azar dermal leishmaniasis is not very uncommon in endemic areas. Our survey showed that kala-azar was verging on a greater proportions and appearance of such an outbreak is serious because of the risk of spread to other areas. In view of the outbreak of kalaazar in Dararhat, it is essential for the health authorities to take immediate measures to control the outbreak and prevent its spread to neighboring villages.

\section{Conclusions:}

Kala -azar is a neglected disease since it affects the neglected sector of our population those who have no voice to spell their basic human rights. The outbreak of kala-azar in that community was outnumbered the usual pattern of distribution of cases. It is obvious that large number of PKDL acts as reservoir of kala-azar. So epidemiological survey and elimination of this chronic neglected disease is necessary.

\section{Conflict of interest: None.}

\section{References:}

1. Sengupta PC. History of kala-azar in India. Indian Med Gaz 1947;82:281-6.

2. Sanyal RK. Leishmaniasis in the Indian sub-continent. In: Chang KP, Bray RS, eds. Leishmaniasis. Amsterdam: Elsevier Science Publishers, B.V., 1985,p.443-67.

3. Birley MH. An historical review of malaria, kala-azar and filariasis in Bangladesh in relation to the Flood Action Plan. Ann Trop Med Parasitol 1993;87:319-34. https://doi.org/ $10.1080 / 00034983.1993 .11812776$
4. Matlashewski G, Arana B, Kroeger A et al. Visceral leishmaniasis: elimination with existing interventions. The Lancet infectious diseases 2011;11(4):322-325. https:// doi.org/10.1016/S1473-3099(10)70320-0

5. Srivastava P, Dayama A, Mehrotra S et al. Diagnosis of visceral leishmaniasis. Transactions of the Royal Society of Tropical Medicine and Hygiene 2011;105(1):1-6. https:// doi.org/10.1016/j.trstmh.2010.09.006

6. Argaw D, Mulugeta A, Herrero M, et al. 2013. Risk Factors for Visceral Leishmaniasis among Residents and Migrants in Kafta-Humera, Ethiopia. PLoS neglected tropical diseases 2013;7(11):2543. https://doi.org/10.1371/journal.pntd. 0002543

7. Bern $\mathrm{C}$ and Chowdhury R. The epidemiology of visceral leishmaniasis in Bangladesh: prospect for improved control. Indian J Med Res 2006;123:275-288.

8. Elias M, Rahman AJ, Khan NI. Visceral leishmaniasis and its control in Bangladesh. Bull World Health Organ 1989;67:439.

9. Rahman KM, Islam N. Resurgence of visceral leishmaniasis in Bangladesh. Bull World Health Organ 1983;61:113-6.

10. National Guideline for Kala-azar Case Management. Kalaazar Elimination Program; Communicable Disease Control (CDC); Directorate General of Health Services; Ministry of Health and Family Welfare, Government of the People's Republic of Bangladesh. May, 2013.

11. Napier LE. The principles and practice of tropical medicine. New York, The Macmillan Co. 1946, p. 134.

12. Wilcocks C \& Manson-Bahr PEC. Manson's tropical diseases, 7th ed. Baltimore, Williams, 1972:123-130.

13. umar R, Das VNR, Topno RK et al. 2016: Para-kala-azar dermal Leishmaniasis cases in Indian subcontinent - A case series. Pathogens and Global Health 2016;110(7-8):326-329. https://doi.org/10.1080/20477724.2016.1258163 